\title{
Comparison of intraocular pressure and hemodynamic responses to insertion of laryngeal mask airway or endotracheal tube in elective paediatric ophthalmic procedures
}

\author{
Swetha Ramani ${ }^{1}$, Radha Krishnan ${ }^{2, *}$, Kala Balasubramaniam ${ }^{3}$, Brinda $^{4}$, Pushparani ${ }^{5}$ \\ ${ }^{1}$ Assistant Professor, Dept. of Anaesthesiology, SRM Medical College and Research Institute, Potheri, Chennai, Tamil Nadu, \\ ${ }^{2}$ Assistant Professor, Dept. of Anesthesiology, Chengalpattu Medical College, Chengalpattu, Chennai, Tamil Nadu, \\ ${ }^{3}$ Professor, Dept. of Anesthesiology, Balaji Medical College, Chennai, Tamil Nadu, ${ }^{4}$ Senior Resident, Dept. of Anesthesiology, \\ Regional Institute of Ophthalmology, Chennai, Tamil Nadu, ${ }^{5}$ Associate Professor, Dept. of Anesthesiology, SRM Medical \\ College and Research Institute, Potheri, Chennai, Tamil Nadu, India
}

*Corresponding Author:

Email: swemohan@gmail.com

\begin{abstract}
Introduction: The main objective of the study was to assess the benefits of using laryngeal mask airway in place of endotracheal intubation in reducing the intraocular pressure and hemodynamic response in paediatric patients posted for elective ophthalmic procedures. This prospective randomized study was conducted over a period of six months in the Regional institute of Ophthalmology.

Materials and Methods: Sixty paediatric patients aged between 2-12 years, belonging to ASA grade $1 \& 2$ and posted for elective ophthalmic procedures were allocated into two groups, each of 30 patients, by a closed envelope method. Laryngeal mask airway (LMA) insertion for group I and Endotracheal intubation (ETI) for group II were performed. Intraocular pressure measurements at Baseline, pre-insertion, at $30^{\text {th }}$ second and at $2^{\text {nd }}$ minutes between the groups were compared. Corresponding responses in heart rate (HR), and mean arterial pressure (MAP) were also compared.

Results: Statistically significant differences were seen at $30^{\text {th }}$ second of intubation between group I and group II in HR [107.8 \pm 13.417 in group I and $126.2 \pm 10.996$ in group II], MAP [84.8 \pm 4.637 in group I and $98.4 \pm 8.6$ in group II] and IOP $(11.07 \pm 1.9 \mathrm{mmHg}$ in group I and $15.76 \pm 2.7 \mathrm{mmHg}$ in group II; $P=0.0001)]$. Similarly, significant differences were also observed at $2^{\text {nd }}$ minute of intubation.

Conclusion: We found that LMA insertion has less influence on intraocular pressure and hemodynamic response and it is associated with less postoperative complications in children undergoing elective ophthalmic procedures under general anaesthesia.
\end{abstract}

Keywords: Laryngeal mask airway, Intubation, Pressor response, Ophthalmic, Intraocular.

\section{Introduction}

The primary considerations in anaesthetic management of ophthalmic surgeries are: An immobile eye with extraocular muscle akinesia, control of the airway with adequate ventilation, haemodynamic stability and well controlled intraocular pressure by avoiding rise in the central venous pressure prior to, during and after the surgery.

Almost always general anaesthesia is required for children undergoing ophthalmic procedures, because they do not co-operate for local anaesthetic blocks as adults do. ${ }^{1}$ But associated stress response to laryngoscopy and intubation in general anesthesia, also increases the intraocular pressure. ${ }^{2,3}$ When the IOP is further increased, the intraocular contents may be forced through the surgical incision which may result in prolapse of iris or lens, expulsive choroidal haemorrhage or vitreous loss. Similarly, any further rise in IOP will lead to permanent loss of vision especially in glaucoma corrective procedures and in repair of penetrating eye injuries. These conditions present special challenges to the anaesthetist and hence, proper anaesthetic management contributes significantly to a successful surgical outcome. ${ }^{4}$

Recently, laryngeal mask airway (LMA) has replaced the conventional endotracheal intubation in paediatric patients undergoing elective procedures. ${ }^{5}$ There are many reports regarding its efficiency in not raising the pressor response, and not raising, as it does not involve laryngoscopy and direct tracheal stimulation. ${ }^{9,10}$ Hence, it is useful for children undergoing ophthalmic surgery, with raised intraocular pressure. There are numerous studies to indicate that the use of LMA has less increase in IOP, blood pressure and heart rate in adult ophthalmic procedures, but few such studies are done in paediatric patients. ${ }^{14,15}$

Hence, this study was under taken to compare the effects of LMA insertion with endotracheal tube intubation on IOP and pressor response and to find a technique which is associated with minimal changes.

\section{Materials and Methods}

After obtaining approval from Ethical committee and informed written parental consent, sixty children of ASA grade I \& II scheduled for elective ophthalmic 
surgeries under general anaesthesia were included into the study. Then the patients under study were randomly allocated into two groups namely, Group I and Group II, each consisting of 30 patients. This was a prospective randomized study done over a period of six months. Randomization was done by sealed opaque envelope method. It was also single blinded. LMA insertion in group I and endotracheal intubation in group II were performed.

Paediatric patients aged from 2 to 12 years, with normal IOP, and posted for unilateral ophthalmic surgery under general anaesthesia were included in the study and those patients not satisfying inclusion criteria, posted for emergency surgery, patients with predicted difficult intubation, patients with increased IOP and those having upper respiratory infection, cardiovascular problem, respiratory disease, neck deformities, history of convulsions, and a known allergy to any of the drugs used were excluded from the study.

Preoperative IOP were recorded with Tonopen or Icare in the OPD [Fig. 4]. Standard fasting protocol for paediatric patients was followed. When the paediatric patients were shifted inside the premedication room, venous cannula was secured and Inj. Glycopyrrolate 10 $\mu \mathrm{g} / \mathrm{kg}$ IV, Inj. Ondansetron $0.1 \mathrm{mg} / \mathrm{kg}$ IV and Inj. Fentanyl $2 \mu \mathrm{g} / \mathrm{kg}$ IV was given. Inside the operation theatre, standard monitoring of all the patients included SPO2, ECG and NIBP and the baseline parameters were recorded. Baseline IOP was measured by the attending surgeon using Schiotz tonometer after instilling $4 \%$ topical lignocaine in the non-operating eye. Induction was done with Inj.Propofol $2 \mathrm{mg} / \mathrm{kg}$ followed by intubating dose of muscle relaxant Inj.Atracurium $0.5 \mathrm{mg} / \mathrm{kg}$. Tracheal intubation or LMA placement was done 3 mins thereafter, after achieving adequate depth of anaesthesia which was observed by easy up and down movement of the mandible and absence of response to bilateral jaw thrust. In the LMA group, the airway was secured with appropriate sized LMA, based on the weight of the child; while in the ETT group, patient's airway was secured with an endotracheal tube of appropriate size. The same anaesthesiologist performed endotracheal intubation or placement of LMA in the studied patients.

Thereafter, further IOP measurement was repeated by Schiotz Tonometer 3 mins after induction, 30 secs after LMA or ETT placement and again 2mins after the airway device placement and the hemodynamic changes were recorded continuously. Classic LMA was inserted by standard finger insertion technique and endotracheal tube was inserted using conventional Laryngoscopy. The controlled ventilation was maintained with oxygen and nitrous oxide mixture in the ratio of $1: 1$ with $0.6 \%$ Isoflurane using a Jackson Rees circuit. The secondary outcome measures like the systolic and diastolic blood pressure, mean arterial pressure, heart rate, respiratory rate, and oxygen saturation were monitored continuously throughout the procedure. Finally, residual neuromuscular blockade was reversed with Inj. Neostigmine and Inj. Glycopyrrolate at the end of the procedure, and extubation of LMA or ETT was done after establishment of spontaneous breathing and response to verbal command. During emergence, incidence of complications like cough, strain and holding of breath were noted. The hemodynamic parameters were continuously monitored in the postoperative period. Patients were observed for any complications like laryngospasm, bronchospasm, blood staining of device, stridor, and hoarseness of voice or painful phonation in the post-operative period.

\section{Data Analysis}

To obtain a power of $80 \%$ and confidence interval of $95 \%$, the required sample size in each group was 28 , approximated to 30 . The sample size was computed based on previous studies, keeping changes in intraocular pressure as the primary outcome variable. Data were analyzed using SPSS17.0. All quantitative variables were summarized in terms of descriptive statistics such as mean and SD and median with range. Paired sample $t$ tests were used to compare the variables "IOP before" and "IOP after"." The Student t test and Mann-Whitney U test were used to compare the different mean values between the 2 groups. Twoway analysis of variance was used to determine whether there was statistically significant difference among the 5 -time points in the hemodynamic parameters between the groups. $\mathrm{P}<.05$ was considered statistically significant.

\section{Sample size calculation:}

$$
\begin{aligned}
& n=\frac{z^{2} \times \hat{p}(1-\hat{p})}{\varepsilon^{2}} \\
& n=\text { sample size } \\
& z^{2}=\text { confidence interval (for } 95 \%=1.96 \text { ) } \\
& \hat{p}=\text { population proportion (incidence is } 400 \text { per } \\
& 10,000 \text { population }=0.04 \text { based on inclusion and } \\
& \text { exclusion criteria) } \\
& \varepsilon^{2}=\text { margin of error taken as } 0.05 \\
& =\quad(1.96)^{2} \times 0.04(1-0.04) \\
& =58.9824 \text { (Total } \mathrm{n}=60, \text { Approx. } 30 \text { in each group })
\end{aligned}
$$

\section{Result}

The sixty paediatric patients were randomly allocated into two groups of 30 patients each

Group I - Subjected to LMA insertion

Group II - Subjected to Endotracheal intubation

The study groups were comparable to each other with respect to their age, weight, height and body mass index. In the demographic parameters, there was no statistically significant difference between the study groups. 


\section{Demographic Profile}

Table 1: Patients' demographic parameters in laryngeal mask airway and endotracheal intubation groups

\begin{tabular}{|l|c|c|}
\hline \multicolumn{1}{|c|}{ Variables } & Group I & Group II \\
\hline $\mathrm{N}$ & 30 & 30 \\
\hline Age in years (SE) & $6.4(0.45)$ & $6.55(0.63)$ \\
\hline Weight in Kgs (SE) & $19.36(1.24)$ & $20.53(1.77)$ \\
\hline BMI (SE) & $16.21(0.49)$ & $15.97(0.48)$ \\
\hline Male: Female & $14: 16$ & $17: 13$ \\
\hline
\end{tabular}

\section{SE: Standard error of Mean}

Time taken for successful placement of the device in one attempt was similar in both the groups (group I $15 \mathrm{~s}$ and group II $18 \mathrm{~s}$ ). Three patients in group I required more than one attempt for securing the airway and two patients required change in size of the LMA. Two patients in group II also required a change in size of the endotracheal tube.

Table 2: Intra ocular pressure (student's t-test)

\begin{tabular}{|l|c|c|c|}
\hline \multicolumn{1}{|c|}{ Intervals } & LMA & ETT & P-value \\
\hline Baseline & $11.65 \pm 1.419$ & $12.03 \pm 2.23$ & 0.4342 \\
\hline Preinsertion & $11.25 \pm 1.454$ & $11.753 \pm 2.347$ & 0.3225 \\
\hline 30 seconds & $11.076 \pm 1.959$ & $15.76 \pm 2.749$ & 0.0001 \\
\hline 2 min & $10.57 \pm 1.6028$ & $14.66 \pm 2.629$ & 0.0001 \\
\hline
\end{tabular}

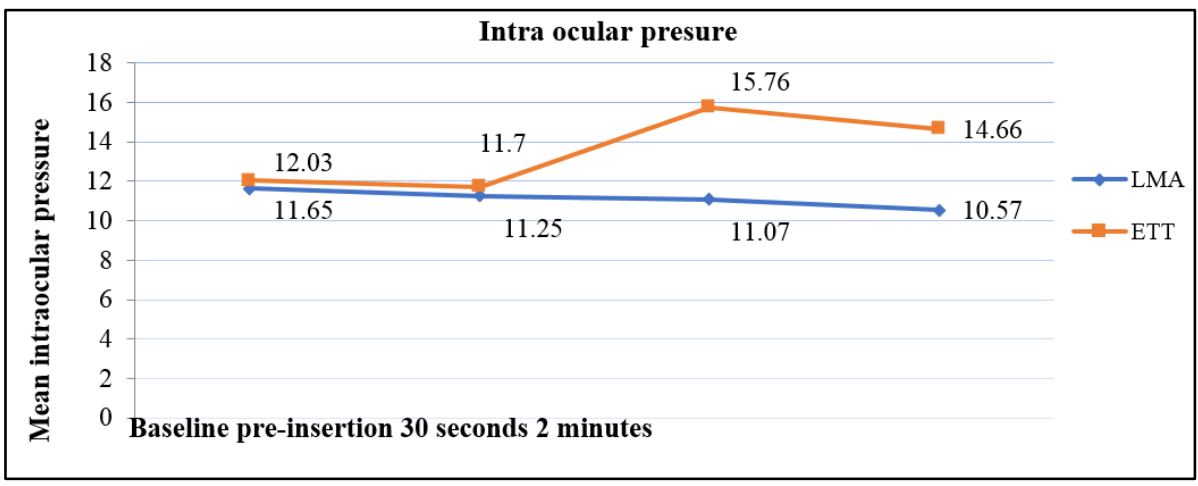

Fig. 1: Intraocular pressure variation in laryngeal mask airway and endotracheal intubation groups $\left({ }^{*} P<\mathbf{0 . 0 5}\right)$

In LMA group the base line IOP was $11.65+1.419 \mathrm{~mm}$ of $\mathrm{Hg}$. There was an initial fall in IOP to $11.25+1.414, \mathrm{~mm}$ of $\mathrm{Hg}$ immediately after induction but before insertion of LMA. It was followed by progressive decline in IOP to $11.076+1.959 \mathrm{~mm}$ of $\mathrm{Hg}$ and to $10.57+1.602 \mathrm{~mm}$ of $\mathrm{Hg}$ at 30 seconds and 2 minutes respectively after insertion of LMA. The fall was highly significant $\mathrm{P}<0.001$.

In ETT group the baseline value of IOP was $12.03+2.23 \mathrm{~mm}$ of $\mathrm{Hg}$. Immediately after induction, but before tracheal intubation, there was a fall in IOP to $11.753+2.347 \mathrm{~mm}$ of $\mathrm{Hg}$. It was followed by a significant rise in IOP at 30 seconds and $2^{\text {nd }}$ minute with mean value of $15.76+2.749 \mathrm{~mm}$ of $\mathrm{Hg}$ and $14.66+2.629 \mathrm{~mm}$ of $\mathrm{Hg}$ respectively. There was a fall in IOP at 2nd minute. But this value was still above the baseline value. The comparison of IOP between both LMA \& ETT group shows that the IOP was significantly raised at 30 seconds and 2 minutes after tracheal intubation $(\mathrm{p}<0.001)$.

Table 3: Mean arterial pressure (student's t-test)

\begin{tabular}{|l|c|c|c|}
\hline \multicolumn{1}{|c|}{ Intervals } & LMA & ETT & P-value \\
\hline Baseline & $82.3 \pm 6.832$ & $82.798 \pm 8.47$ & 0.8030 \\
\hline Preinsertion & $83.5 \pm 4.511$ & $84.52 \pm 9.273$ & 0.5901 \\
\hline 30 seconds & $84.8 \pm 4.637$ & $98.4 \pm 8.6$ & 0.0001 \\
\hline 2 min & $84.2 \pm 4.546$ & $91.7 \pm 4.815$ & 0.0001 \\
\hline
\end{tabular}




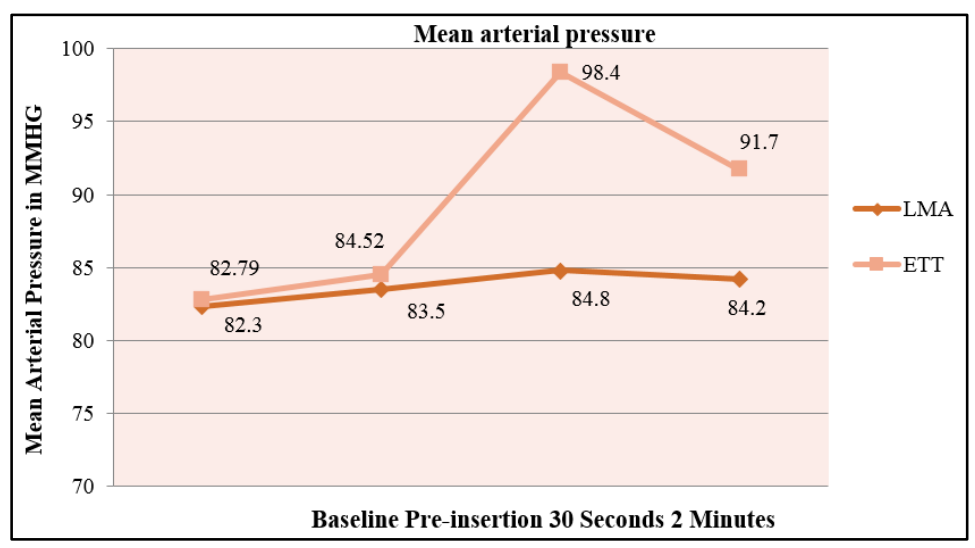

Fig. 2: Mean arterial blood pressure variation in laryngeal mask airway and endotracheal intubation groups $\left({ }^{*} P<0.05\right)$

In the LMA group the mean baseline mean blood pressure (MAP) was $82.3+6.832 \mathrm{~mm}$ of $\mathrm{Hg}$ (mean + S.D). There was a declining trend in MAP after induction and throughout the study. In ETT group, the mean baseline MAP was $82.798+8.47 \mathrm{~mm}$ of $\mathrm{Hg}$ (mean
+ SD). After induction and before intubation, there was an initial fall in blood pressure and was raised after 30seconds of intubation but at 2 nd minute there was a decline in mean value of blood pressure MAP, but the fall was not below the baseline value. $(\mathrm{P}<0.05)$

Table 4: Heart rate (student's t-test):

\begin{tabular}{|l|c|c|c|}
\hline \multicolumn{1}{|c|}{ Intervals } & LMA & ETT & P-value \\
\hline Baseline & $101.33 \pm 11.36$ & $105.1 \pm 12.87$ & 0.2339 \\
\hline Preinsertion & $105.36 \pm 12.22$ & $108.1 \pm 11.795$ & 0.3805 \\
\hline 30 seconds & $107.8 \pm 13.417$ & $126.2 \pm 10.996$ & 0.0001 \\
\hline $2 \mathrm{~min}$ & $104.03 \pm 10.607$ & $117.36 \pm 11.654$ & 0.0001 \\
\hline
\end{tabular}

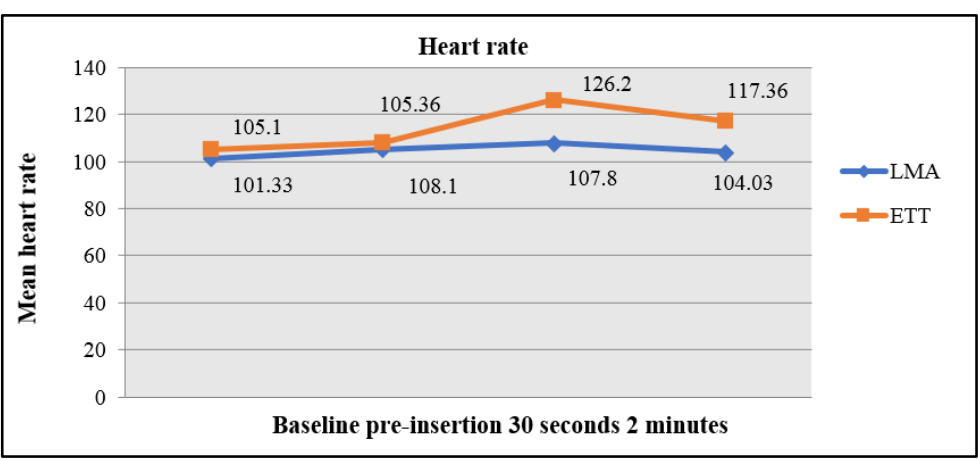

Fig. 3: Heart rate variation in laryngeal mask airway and endotracheal intubation groups $\left({ }^{*} P<0.05\right)$

In LMA group the mean value of $\mathrm{HR}$ was $101.33+11.36 /$ min (mean + S.D). There was a declining trend throughout the procedure. In ETT group the HR was 101.5+12.87/ $\mathrm{min}$ and had a rising trend in subsequent timings. Therefore, there was a rising trend in heart rate in ETT group compared to LMA group. $(\mathrm{P}<0.05)$

Table 5: Complications

\begin{tabular}{|l|c|c|c|}
\hline Complications & LMA & ETT & Incidence \\
\hline Breath Holding & 1 & 5 & $16.667 \%$ \\
\hline Coughing & 1 & 7 & $23.333 \%$ \\
\hline Straining & 1 & 7 & $23.333 \%$ \\
\hline Nil & 27 & 11 & $36.667 \%$ \\
\hline
\end{tabular}

Out of 30 children in ETT group, 7 children had coughing during emergence whereas in LMA group, only one child had coughing. The incidence of straining was $23.33 \%$ in ETT group but only $3.33 \%$ in LMA group. The occurrence of breath-holding during emergence was higher in ETT group $16.6 \%$ but it was $6.6 \%$ in LMA group.

\section{Discussion}

Conventional laryngoscopy with general anaesthesia is usually practiced in paediatric patients coming for Intra-ocular procedure. But laryngoscopy and intubation, stimulates the sympathetic system resulting in increase in IOP, with associated rise in MAP \& HR. ${ }^{6,7}$ This sudden disproportionate increase in 
MAP and HR following laryngoscopy and intubation are predominantly due to stimulation of oropharyngeal and laryngopharyngeal structures eliciting increased sympathetic activity. The distortions of supraglottic structures during laryngoscopy may be the chief stimulus for the increased sympatho-adrenal activity. ${ }^{8}$ LMA insertion do not require laryngoscopy and hence the stimulation of supraglottic structures are less. The LMA, once placed, is located in the hypopharynx, where the anterior surface of the LMA contacts the posterior part of the tongue with less distortion of supraglottic structures. Therefore, the adrenergic response and hence the hemodynamic changes are less during LMA insertion than during the endotracheal intubation. Also, the LMA usage minimizes the associated rise in the IOP. LMA insertion is also considered to produce relatively less trauma to the airway than the conventional endotracheal intubation. ${ }^{9}$

There are numerous studies in adults undergoing ophthalmic surgeries where hemodynamic changes between LMA and ETT have been compared. But there are only few studies conducted in children undergoing ophthalmic surgeries, to compare the effect of insertion of LMA with endotracheal intubation on IOP. Among the few studies in children, two studies were conducted in non-ophthalmic surgeries to compare the effect on IOP. Hence, we conducted our study in paediatric patients undergoing ophthalmic surgery to assess the influence of using LMA over ETT in reducing the hemodynamic response and the IOP changes during the intraoperative period.

In the OPD, the pre-operative IOP was recorded in children using I-care or Tonopen as these require much less co-operation from children [Fig. 4]. The baseline IOP was recorded only after induction with $4 \%$ topical lignocaine drops applied in the non-operating eye alone. By avoiding IOP measurement in the operative eye, inside the operating theatre, we avoid the chance of introducing any new infection into the well prepared operative eye.
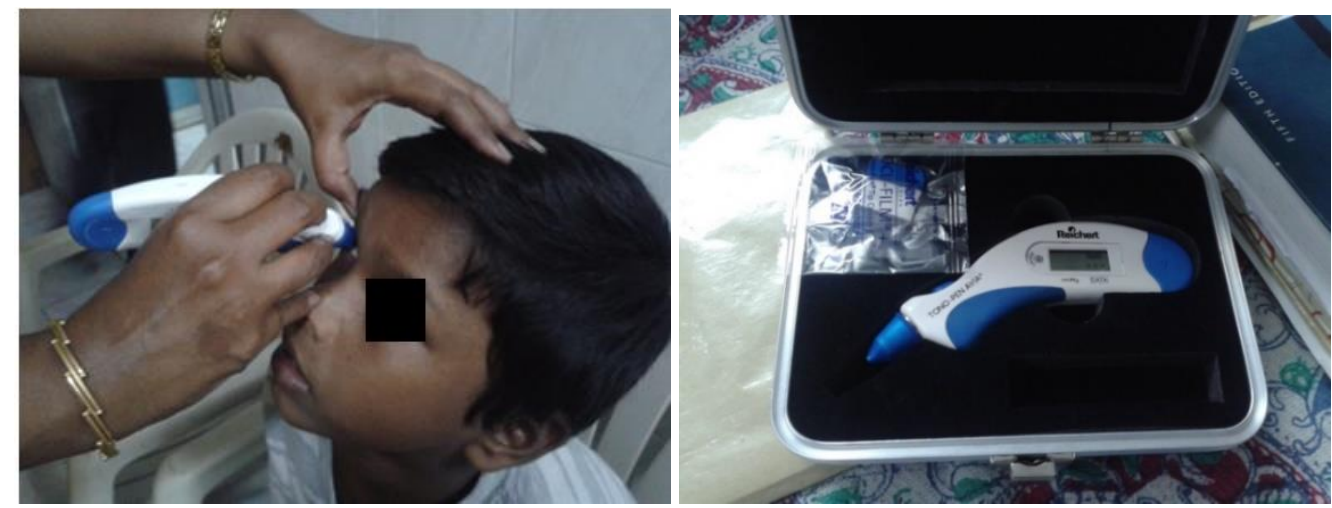

Fig. 4: Photograph showing pre-operative intra ocular pressure measurement using tonopen

The initial simultaneous minimal fall in MAP and HR which was observed in both the study groups, immediately following intravenous induction may be due to adequate depth of anesthesia provided by Propofol [Table 3, Fig. 2]. The induction with Propofol and fentanyl combination gives adequate depth of anesthesia producing this minimal and acceptable fall in hemodynamics. ${ }^{10}$ The rise in mean arterial pressure in the ETT group at $30^{\text {th }}$ second $(98.4 \pm 8.6)$ and $2^{\text {nd }}$ minute $(91.7 \pm 4.81)$ of intubation, was significant. The $30^{\text {th }}$ second HR $(126.2 \pm 10.996)$ and $2^{\text {nd }}$ minute HR (117.36 \pm 11.654$)$ following ETT intubation was also found to be significantly higher when compared to LMA group [Table 4, Fig. 3]. But on the other hand, in the LMA group, there is relatively lesser rise in the mean arterial pressure and the heart rate following its placement. This significant hemodynamic change in the ETT group following intubation can be attributed to a larger sympatho-adrenal response during laryngoscopy and endotracheal intubation.
Although there is a good correlation to rise in IOP with respect to increase in CVP, we did not study this correlation as there was no requirement of invasive monitoring in the selected elective ophthalmic procedures. ${ }^{11,12}$

The rise in IOP with endotracheal intubation is tolerated to some extent in normal eye but may produce deleterious effect in patients with already raised IOP like acute angle closure glaucoma or in children with penetrating eye injury. Even a small rise in IOP for a short time in these patients, may cause optic disc ischaemia, resulting in blindness in acute angle closure glaucoma and in penetrating eye injury, expulsion of intraocular contents may occur with any further rise in IOP. In chronic glaucoma the incidence of loss of central vision is about $30 \%$ when the IOP rises above $20 \mathrm{mmHg}$, whereas the incidence is less when IOP is below $18 \mathrm{mmHg} .^{13}$

In our study, the mean IOP rise was higher in ETT group when compared with LMA group during following airway placement. When compared with 
LMA group, the variation in IOP at different time points was greater in ETT group during the study. In both the study groups, there existed a correlation between the hemodynamic variation with respect to the observed variation in IOP. In most of the cases in our study, tracheal intubation produced a rise in HR and MAP whereas LMA insertion was not associated with rise in these variables.

Barclay et al have done similar randomized prospective study in 20 adult patients with glaucoma to compare the effects of tracheal intubation and laryngeal mask airway insertion on IOP, MAP and HR under general anesthesia. They have concluded that after induction with Propofol, both groups had similar fall in IOP but then there was a continued fall in IOP only in LMA group in contrasts to ETT group where there was an increase in IOP after intubation. This rise was accompanied by increase in HR and MAP in ETT group. Similar observations have been noted in our study. ${ }^{14}$

40 patients were analysed for changes in IOP by Myint et al during spontaneous ventilation with LMA and controlled ventilation using tracheal tube, undergoing intraocular surgery. They also have used Propofol as an induction agent along with enflurane, N20 in oxygen. The IOP was lower than the baseline \& similar in both the groups. After extubation, the IOP was significantly higher than LMA group. ${ }^{15}$ Our study was done with controlled ventilation in both the groups. The IOP values were raised after tracheal intubation and not with LMA insertion.

Bukari et al conducted a prospective randomized study on fifty adult patients undergoing non-ophthalmic surgeries and concluded that the increase in $\mathrm{HR}$ in LMA \& ETT group started immediately after intubation and it remained raised for more prolonged time in ETT group compared to LMA group. Also, in their study, the increase in blood pressure in ETT group at $1^{\text {st }}$ and $2^{\text {nd }}$ minute of intubation, was significantly higher in ETT group. The IOP measurement showed an initial fall in both the study groups but then there was subsequent raise in IOP in ETT group alone. ${ }^{16}$

Coughing, straining, breath-holding, raises intraocular pressure. Coughing may increase IOP up till $50 \mathrm{mmHg}$. In our study, the incidence of coughing during emergence was high among children who were intubated with ETT rather than with LMA. During extubation, LMA offers smooth recovery required for ophthalmic procedures with minimal coughing as against extubation of ETT. ${ }^{17,18}$ Out of 30 children in ETT group, 7 children had coughing during emergence whereas in LMA group, only one child had coughing. The incidence of straining was $23.33 \%$ in ETT group but was only 3.33\% in LMA group [Table 5].

There was 4- fold increase in the incidence of breath-holding during emergence in ETT group (16.66\%) than in the LMA group. In both groups, none of the children reported post-operative sore throat. The overall statistical analysis of the present study and most of the literature published reveals that there is significant increase in heart rate, mean blood pressure and IOP in the ETT group when compared to the LMA group.

Abhiruchi Patki have concluded in his metaanalysis of sixteen prospective randomized controlled studies assessing the advantage of LMA over ETT intubation in paediatric age group that there is lower incidence of coughing during emergence and less sore throat incidence in the LMA group compared to ETT intubation. ${ }^{17}$ Kilickan L, Baykara N, et al have reported high incidence of coughing and straining during emergence in tracheally intubated adult patients. ${ }^{18}$ Denny and co-workers have also reported similar findings in adult patients. The incidence of breath holding was higher in ETT group than in the LMA group. ${ }^{19}$ In our study, the occurrence of breath-holding during emergence was higher in ETT group $16.6 \%$ versus $6.6 \%$ in LMA group.

\section{Limitation}

The gold standard technique of measuring IOP is Applanation tonometry not the Schiotz tonometer. Since the ophthalmic surgeons in our institute, traditionally used the Schiotz tonometer for measuring intraoperative IOP, we used the same for our study purpose. This was the only limitation in our study.

There are no controversies raised by this study. Since there are limited studies in paediatric ophthalmic anesthesia, we suggest more such studies should be conducted in future.

\section{Conclusion}

From our study, we conclude that this study is in favour of use of LMA to a conventional use of endotracheal intubation for administrating general anaesthesia for intraocular surgeries in children. It has less influence on intraocular pressure and hemodynamics. Reusable LMA is cost effective when compared to endotracheal tube. The LMA could be useful in situations where minimal changes in hemodynamic and intraocular pressure are desirable like cardiac disease and glaucoma. The art of placing an LMA in children can be learnt easily and airway can be secured with confidence.

\section{Conflict of Interest: None}

\section{References}

1. Rodgers A, Cox RG. Anesthetic management for pediatric strabismus surgery: Continuing professional development. Can J Anaesth. 2010;57(6):602-17.

2. Bishop MJ, Bedford RF, Kil HK. Physiologic and Pathophysiologic Responses to Intubation. Anesthesiology Clinics of North America. 1995;13(2):361-375. 
3. Cunningham AJ, Barry P. Intraocular pressure-physiology and implications for anaesthetic management. Can AnaesthSoc J. 1986;33(2):195-208.

4. Harry Murgatroyd, Jane Bembridge, Intraocular pressure. Oxford Journals, CEACCP. 2008;8(3).

5. Bhavesh Patel, Robert Bingham. Laryngeal mask airway and other supraglottic airway devices in paediatric practice. Continuing Education in Anaesthesia, Critical Care \& Pain. 2009;9(1).

6. Shribman AJ, Smith G, Achola KJ. Cardiovascular and catecholamine responses to laryngoscopy with and without tracheal intubation. Br J Anaesth. 1987;59:295-9.

7. Derbyshire DR, Chmielewski A, Fell D, Vater M. Plasma catecholamine response to tracheal intubation. $\mathrm{Br} \mathrm{J}$ Anaesth. 1983;55:855-60

8. Low JM, Harvey JT. Haemodynamic consequences of ind uction and endotracheal intubation. British Journal of Anaesthesia. 1986;58:471-477

9. R. Holden, C.D.G Morsman, J. Butler. Intraocular pressure changes using the laryngeal mask airway and tracheal tube. Anesthesia. 1991;46:922-924

10. Mohsen Ziyaeifard, Rasoul Azarfarin. A comparison of intraocular pressure and hemodynamic responses to insertion of laryngeal mask airway or endotracheal tube using anesthesia with propofol and remifentanil in cataract surgery. J Res Med Sci. 2012;17(6):503-507.

11. Macri FJ. Vascular pressure relationships and the intraocular pressure. Arch Opthalmol. 1961;65:571-4.

12. Murphy DF. Anesthesia and intraocular pressure. Anesth Analg. 1985;64:520-30.

13. Paletta Guedes RA, Paletta Guedes VM. Anesthesia for Glaucoma Surgery. J Fr Ophtalmol. 2009;32(3):221-5.

14. Barclay K, Wakk T, Wareham K. Intraocular pressure ch anges in patients with glaucoma. Comparison between the laryngeal mask airway tracheal tube. Anesthesia. 1994;49(2):159-62.
15. Myint Y, Sing AK, Peacock JE. Changes in intraocular pressure during general anesthesia. A comparison of spontaneous breathing through laryngeal mask ventilation through a tracheal tub e. Anesthesia. 1995;50(2):1269.

16. Bukhari, SA. Pressor Responses and Intraocular Pressure Changes Following Insertion of Laryngeal Mask Airway: Comparison with Tracheal Tube Insertion. Indian J Anaesth. 2003;47(6):473-475.

17. Abhiruchi Patki. Laryngeal mask airway vs the endotracheal tube in paediatric airway management: A meta-analysis of prospective randomised controlled trials. Indian J Anaesth. 2011;55(5):537-541.

18. Kilickan L, Baykara N. The effect on intraocular of endotracheal intubation or laryngeal mask airway use during TIVA without the use of muscle relaxants. Acta Anaesthesiol Scand. 1999;43:343-346.

19. Denny NM. Complications following general anaesthesia for cataract surgery: a comparison of the laryngeal mask airway with tracheal intubation. Journal of the Royal Society of Medicine. 1993;86:521.

How to cite this article: Ramani S, Krishnan R, Balasubramaniam K, Brinda, Pushparani. Comparison of intraocular pressure and hemodynamic responses to insertion of laryngeal mask airway or endotracheal tube in elective paediatric ophthalmic procedures. Indian J Clin Anaesth. 2018;5(3):334-340. 